\title{
Existence of Positive Solutions to Boundary Value Problem of Caputo Fractional Differential Equation
}

\author{
Yaqiong Cui, ${ }^{1}$ Shugui Kang, ${ }^{1}$ and Zhiping Liu ${ }^{2}$ \\ ${ }^{1}$ School of Mathematics and Computer Science, Shanxi Datong University, Datong 037009, China \\ ${ }^{2}$ School of Science, North University, Taiyuan 030051, China \\ Correspondence should be addressed to Shugui Kang; dtkangshugui@126.com
}

Received 8 October 2015; Accepted 17 November 2015

Academic Editor: Chris Goodrich

Copyright (c) 2015 Yaqiong Cui et al. This is an open access article distributed under the Creative Commons Attribution License, which permits unrestricted use, distribution, and reproduction in any medium, provided the original work is properly cited.

We consider the existence of positive solutions to the nonlinear fractional differential equation boundary value problem ${ }^{C} D_{0+}^{\alpha} u(t)+$ $f\left(u(t), u^{\prime}(t)\right)=0, t \in(0,1), u(0)=u(1)=u^{\prime \prime}(0)=0$, where $f:[0,+\infty) \times \mathbb{R} \rightarrow[0,+\infty)$ is continuous, $\alpha \in(2,3]$, and ${ }^{C} D_{0+}^{\alpha}$ is the standard Caputo differentiation. By using fixed point theorems on cone, we give some existence results concerning positive solutions. Here the solutions especially are the interior points of cone.

\section{Introduction}

In this paper, we consider the existence of positive solutions to the following nonlinear fractional differential equation boundary value problem (BVP):

$$
\begin{aligned}
{ }^{C} D_{0+}^{\alpha} u(t)+f\left(u(t), u^{\prime}(t)\right) & =0, \quad t \in(0,1), \\
u(0) & =u(1)=u^{\prime \prime}(0)=0,
\end{aligned}
$$

where $f:[0,+\infty) \times \mathbb{R} \rightarrow[0,+\infty)$ is continuous, $\alpha \in(2,3]$, and ${ }^{C} D_{0+}^{\alpha}$ is the standard Caputo differentiation.

Owing to the rapid development of the theory of fractional calculus itself as well as its applications, fractional differential equations have attracted intensive study recently. There has been especially an increased interest in studying the existence of positive solutions for the continuous fractional calculus concerning the Riemann-Liouville and Caputo derivatives; see [1-7] and references therein. For example, if $\alpha \in(1,2]$, using the cone expansion or the cone compression fixed point theorem, Bai and Lü [1] studied the existence of positive solutions. If $\alpha \in(3,4]$, by the similar methods as [1], Xu et al. [2] obtained the existence of multiple positive solutions. As far as we know, there are many papers of fractional order which have allowed the boundary value conditions to depend on $u^{\prime}$; see [2-6]. However, to the authors' knowledge, if $\alpha>2$, there are few papers of fractional order subjected to the boundary value conditions where the first order $u^{\prime}$ is not involved. Motivated by the above results and [8-10], to cover up this gap, if $\alpha \in(2,3]$, we mainly discuss the existence of positive solutions to fractional differential equation which is under the boundary value conditions $u(0)=u(1)=u^{\prime \prime}(0)=0$. In our paper, we firstly derive the corresponding Green's function which is different from these Green's functions that appeared in the references here and give some properties. Finally, based on Schauder's fixed point theorem, the cone expansion or the cone compression fixed point theorem, and an extension of Krasnoselskii's fixed point theorem, we obtain the existence of positive solutions and give some examples to illustrate our results. Here the solutions especially are the interior points of cone; thus the solutions have better properties.

\section{Preliminary}

In this section (refer to $[11,12]$ ), we list some necessary notations, lemmas, and theorems.

Definition 1 (see [12]). The Caputo fractional derivative of order $\alpha>0$ of a continuous function $u:(0,+\infty) \rightarrow \mathbb{R}$ is given by

$$
{ }^{C} D_{0+}^{\alpha} u(t)=\frac{1}{\Gamma(n-\alpha)} \int_{0}^{t} \frac{u^{(n)}(s)}{(t-s)^{\alpha-n+1}} d s,
$$


where $n=[\alpha]+1$ and $[\alpha]$ denotes the integer part of the real number $\alpha$, provided that the right side is pointwise defined on $(0,+\infty)$.

Lemma 2 (see [12]). Let $\alpha>0$; if $u \in A C^{n}[0,1]$ or $u \in$ $C^{n}[0,1]$, then

$$
I_{0+}^{\alpha}{ }^{C} D_{0+}^{\alpha} u(t)=u(t)-\sum_{k=0}^{n-1} \frac{u^{(k)}(0)}{k !} t^{k},
$$

where $n=[\alpha]+1$ and $I_{0+}^{\alpha}$ denotes the Riemann-Liouville fractional integral of order $\alpha$.

Lemma 3. Let $\alpha \in(2,3]$. Given $h \in C[0,1]$, the unique solution of

$$
\begin{aligned}
{ }^{C} D_{0+}^{\alpha} u(t)+h(t) & =0, \quad t \in(0,1), \\
u(0) & =u(1)=u^{\prime \prime}(0)=0
\end{aligned}
$$

is

$$
u(t)=\int_{0}^{1} G(t, s) h(s) d s
$$

here

$$
G(t, s)= \begin{cases}\frac{t(1-s)^{\alpha-1}-(t-s)^{\alpha-1}}{\Gamma(\alpha)}, & 0 \leqslant s \leqslant t \leqslant 1, \\ \frac{t(1-s)^{\alpha-1}}{\Gamma(\alpha)}, & 0 \leqslant t \leqslant s \leqslant 1\end{cases}
$$

is Green's function of (4)-(5).

Proof. We apply Lemma 2 to reduce (4) to an equivalent equation,

$$
u(t)=-I_{0+}^{\alpha} h(t)+C_{1}+C_{2} t+C_{3} t^{2},
$$

for some $C_{1}, C_{2}, C_{3} \in \mathbb{R}$. Then

$$
\begin{aligned}
& u^{\prime}(t)=-I_{0+}^{\alpha-1} h(t)+C_{2}+2 C_{3} t, \\
& u^{\prime \prime}(t)=-I_{0+}^{\alpha-2} h(t)+2 C_{3} .
\end{aligned}
$$

By (5) and the above equalities, we get $C_{1}=C_{3}=0$ and

$$
C_{2}=\frac{1}{\Gamma(\alpha)} \int_{0}^{1}(1-s)^{\alpha-1} h(s) d s .
$$

Therefore, the unique solution of problem (4)-(5) is

$$
\begin{aligned}
u(t)= & \frac{-1}{\Gamma(\alpha)} \int_{0}^{t}(t-s)^{\alpha-1} h(s) d s \\
& +\frac{t}{\Gamma(\alpha)} \int_{0}^{1}(1-s)^{\alpha-1} h(s) d s \\
= & \frac{1}{\Gamma(\alpha)} \int_{0}^{t}\left[t(1-s)^{\alpha-1}-(t-s)^{\alpha-1}\right] h(s) d s \\
& +\frac{t}{\Gamma(\alpha)} \int_{t}^{1}(1-s)^{\alpha-1} h(s) d s \\
= & \int_{0}^{1} G(t, s) h(s) d s .
\end{aligned}
$$

The proof is completed.
Lemma 4. $G(t, s)$ has following properties:

(i) $G(t, s) \in C([0,1] \times[0,1], \mathbb{R})$, and $G(t, s)>0, t, s \in$ $(0,1)$;

(ii) $\left(t-t^{\alpha-1}\right)(1-s)^{\alpha-1} \leqslant \Gamma(\alpha) G(t, s) \leqslant(1-s)^{\alpha-1}, t, s \in$ $[0,1]$.

Proof. Since $\alpha \in(2,3]$, then

$$
\begin{gathered}
t(1-s)^{\alpha-1}-(t-s)^{\alpha-1} \geqslant t(1-s)^{\alpha-1}-(t-t s)^{\alpha-1} \\
\quad=\left(t-t^{\alpha-1}\right)(1-s)^{\alpha-1}>0, \quad t, s \in(0,1)
\end{gathered}
$$

this implies that properties (i) and (ii) hold. The proof is completed.

The following fixed point theorems are fundamental in the proofs of our main results.

Theorem 5 (see [11]). Let $E$ be a real Banach space, $P \subset E a$ cone, and $\Omega_{1}, \Omega_{2}$ two bounded open subsets of E centered at the origin with $\bar{\Omega}_{1} \subset \Omega_{2}$. Assume that $A: P \cap\left(\bar{\Omega}_{2} \backslash \Omega_{1}\right) \rightarrow P$ is a completely continuous operator such that either of the following holds:

(i) $\|A u\| \leqslant\|u\|, u \in P \cap \partial \Omega_{1}$ and $\|A u\| \geqslant\|u\|, u \in P \cap$ $\partial \Omega_{2}$,

(ii) $\|A u\| \geqslant\|u\|, u \in P \cap \partial \Omega_{1}$ and $\|A u\| \leqslant\|u\|, u \in P \cap$ $\partial \Omega_{2}$.

Then $A$ has at least one fixed point in $P \cap\left(\bar{\Omega}_{2} \backslash \Omega_{1}\right)$.

Let $E$ be a real Banach space and $P \subset E$ a cone. Suppose $\alpha, \beta: E \rightarrow \mathbb{R}^{+}$are two continuous convex functionals satisfying

$$
\begin{aligned}
& \alpha(\lambda u)=|\lambda| \alpha(u), \\
& \beta(\lambda u)=|\lambda| \beta(u),
\end{aligned}
$$

$u \in E, \lambda \in \mathbb{R}$

and there exists a constant $k>0$ such that

$$
\|u\| \leqslant k \max \{\alpha(u), \beta(u)\}, \quad u \in E,
$$

and $\alpha\left(u_{1}\right) \leqslant \alpha\left(u_{2}\right), u_{1}, u_{2} \in P$, and $u_{1} \leqslant u_{2}$.

Theorem 6 (see [10, Theorem 2.1]). Let $r_{2}>r_{1}>0, L>0$ be constants and $\Omega_{i}=\left\{u \in E: \alpha(u)<r_{i}, \beta(u)<L\right\}(i=1,2)$ two bounded open sets in $E$. Let $D_{i}=\left\{u \in E: \alpha(u)=r_{i}\right\}(i=$ $1,2)$. Assume $A: P \rightarrow P$ is a completely continuous operator satisfying

$\left(B_{1}\right) \alpha(A u)<r_{1}, u \in D_{1} \cap P ; \alpha(A u)>r_{2}, u \in D_{2} \cap P$;

$\left(B_{2}\right) \beta(A u)<L, u \in P$;

$\left(B_{3}\right)$ there is a $w \in\left(\Omega_{2} \cap P\right) \backslash\{\theta\}$ such that $\alpha(w) \neq 0$ and $\alpha(u+\lambda w) \geqslant \alpha(u), u \in P, \lambda \geqslant 0$.

Then $A$ has at least one fixed point in $\left(\Omega_{2} \backslash \bar{\Omega}_{1}\right) \cap P$. 
From now on, we assume

$$
E=\left\{u \in C^{1}[0,1]: u(0)=u(1)=0\right\} .
$$

It is well known that $\|u\|_{0} \leqslant\left\|u^{\prime}\right\|_{0}$ for all $u \in E$, where $\|u\|_{0}=$ $\max _{t \in[0,1]}|u(t)|$. Define functionals $\alpha(u)=\|u\|_{0}, \beta(u)=\left\|u^{\prime}\right\|_{0}$, $u \in E$; then $E$ is a real Banach space with the equivalent norm $\|u\|=\beta(u)$ and $\|u\| \leqslant \max \{\alpha(u), \beta(u)\}$.

Define operators $A, K$, and $F$, respectively, by

$$
\begin{aligned}
& K u(t)=\int_{0}^{1} G(t, s) u(s) d s, \\
& F u(t)=f\left(u(t), u^{\prime}(t)\right),
\end{aligned}
$$

$$
t \in[0,1], u \in E
$$

and $A=K F$. It is clear that the solution of BVP (1) is equivalent to the fixed point of $A$ in $E$. We will find the nonzero fixed point of $A$ by using the fixed point theory in cone. For this, we choose cone $P$ of $E$ by

$$
P=\left\{u \in E: u(t) \geqslant \gamma\|u\|_{0}, t \in\left[\frac{1}{4}, \frac{3}{4}\right]\right\},
$$

where

$$
\gamma=\min _{1 / 4 \leqslant t \leqslant 3 / 4}\left(t-t^{\alpha-1}\right)
$$

Lemma 7. Assume $f:[0,+\infty) \times \mathbb{R} \rightarrow[0,+\infty)$ is continuous. Then $A: P \rightarrow P(E \rightarrow E)$ is completely continuous.

Proof. For $u \in P$, from the second inequality of property (ii) of Lemma 4, we have

$$
\|A u\|_{0} \leqslant \frac{1}{\Gamma(\alpha)} \int_{0}^{1}(1-s)^{\alpha-1} F u(s) d s
$$

From (19) and the first inequality of property (ii) of Lemma 4, we have

$$
\begin{aligned}
A u(t) & \geqslant \frac{1}{\Gamma(\alpha)} \int_{0}^{1}\left(t-t^{\alpha-1}\right)(1-s)^{\alpha-1} F u(s) d s \\
& \geqslant\left(t-t^{\alpha-1}\right)\|A u\|_{0}, \quad t \in[0,1] ;
\end{aligned}
$$

then

$$
A u(t) \geqslant \gamma\|A u\|_{0}, \quad t \in\left[\frac{1}{4}, \frac{3}{4}\right] .
$$

It follows from Lemma 3 that

$$
A u(t)=\frac{\int_{0}^{t}\left[t(1-s)^{\alpha-1}-(t-s)^{\alpha-1}\right] F u(s) d s+\int_{t}^{1} t(1-s)^{\alpha-1} F u(s) d s}{\Gamma(\alpha)} .
$$

By direct calculation, we have

$$
(A u)^{\prime}(t)=\frac{\int_{0}^{t}\left[(1-s)^{\alpha-1}-(\alpha-1)(t-s)^{\alpha-2}\right] F u(s) d s+\int_{t}^{1}(1-s)^{\alpha-1} F u(s) d s}{\Gamma(\alpha)} .
$$

Since $f:[0,+\infty) \times \mathbb{R} \rightarrow[0,+\infty)$ is continuous, it is easy to see from (21)-(23) and Lemma 4 that $A: P \rightarrow P$ is continuous. Now, we only need to show that $A$ is compact. Let $\Omega \subset P$ be bounded; that is, there exists a constant number $M>0$ such that $\|u\| \leqslant M$ for $u \in \Omega$. By the definition of $\|u\|$, we know $0 \leqslant u(t),\left|u^{\prime}(t)\right| \leqslant M, u \in \Omega$. Let $C=$ $\max _{0 \leqslant u,|v| \leqslant M} f(u, v)$. Then for $u \in \Omega$, by (19), we have

$$
|A u(t)| \leqslant \frac{C}{\Gamma(\alpha)} \int_{0}^{1}(1-s)^{\alpha-1} d s=\frac{C}{\alpha \Gamma(\alpha)},{ }^{t \in[0,1] .}
$$

By (23) and Lemma 4, we have

$$
\begin{array}{r}
\left|(A u)^{\prime}(t)\right| \leqslant \frac{C}{\Gamma(\alpha)} \int_{0}^{1} 2(1-s)^{\alpha-2} d s=\frac{2 C}{(\alpha-1) \Gamma(\alpha)}, \\
t \in[0,1]
\end{array}
$$

thus $A(\Omega)$ is bounded. Let $t_{1}, t_{2} \in[0,1]$ with $t_{1} \leqslant t_{2}$, for $u \in \Omega$; we have

$$
\begin{aligned}
& \left|A u\left(t_{2}\right)-A u\left(t_{1}\right)\right| \leqslant \int_{0}^{t_{1}}\left|G\left(t_{2}, s\right)-G\left(t_{1}, s\right)\right| \\
& \cdot F u(s) d s+\int_{t_{2}}^{1}\left|G\left(t_{2}, s\right)-G\left(t_{1}, s\right)\right| F u(s) d s \\
& +\int_{t_{1}}^{t_{2}}\left|G\left(t_{2}, s\right)-G\left(t_{1}, s\right)\right| F u(s) d s<\frac{C}{\Gamma(\alpha)} \\
& \cdot \int_{0}^{t_{1}}\left[\left(t_{2}-t_{1}\right)(1-s)^{\alpha-1}+\left(t_{2}-s\right)^{\alpha-1}\right. \\
& \left.-\left(t_{1}-s\right)^{\alpha-1}\right] d s
\end{aligned}
$$




$$
\begin{aligned}
& +\frac{C}{\Gamma(\alpha)}\left(\int_{t_{2}}^{1}\left(t_{2}-t_{1}\right)(1-s)^{\alpha-1} d s\right. \\
& \left.+\int_{t_{1}}^{t_{2}}\left[\left(t_{2}-t_{1}\right)(1-s)^{\alpha-1}+\left(t_{2}-s\right)^{\alpha-1}\right] d s\right) \\
& =\frac{C\left(t_{2}-t_{1}\right)}{\Gamma(\alpha)} \int_{0}^{1}(1-s)^{\alpha-1} d s \\
& +\frac{C}{\Gamma(\alpha)}\left[\int_{0}^{t_{1}}\left[\left(t_{2}-s\right)^{\alpha-1}-\left(t_{1}-s\right)^{\alpha-1}\right] d s\right. \\
& \left.+\int_{t_{1}}^{t_{2}}\left(t_{2}-s\right)^{\alpha-1} d s\right] \leqslant \frac{C}{\alpha \Gamma(\alpha)}\left(t_{2}-t_{1}+t_{2}^{\alpha}-t_{1}^{\alpha}\right), \\
& \left|(A u)^{\prime}\left(t_{2}\right)-(A u)^{\prime}\left(t_{1}\right)\right| \\
& \quad \leqslant \frac{C}{\Gamma(\alpha-1)}\left(\int_{0}^{t_{1}}\left[\left(t_{2}-s\right)^{\alpha-2}-\left(t_{1}-s\right)^{\alpha-2}\right] d s\right. \\
& \left.+\int_{t_{1}}^{t_{2}}\left(t_{2}-s\right)^{\alpha-2} d s\right) \leqslant \frac{C}{\Gamma(\alpha)}\left(t_{2}^{\alpha-1}-t_{1}^{\alpha-1}\right) .
\end{aligned}
$$

The Arzela-Ascoli theorem guarantees that $A(\Omega)$ is relatively compact, which means $A$ is compact. Hence $A: P \rightarrow$ $P(E \rightarrow E)$ is completely continuous. The proof is completed.

Lemma 8. Assume $f:[0,+\infty) \times \mathbb{R} \rightarrow[0,+\infty)$ is continuous. If $u \in P \backslash\{\theta\}$ is the solution of $B V P(1)$, then $u \in P^{o}$.

Proof. If $u \in P \backslash\{\theta\}$ is the solution of BVP (1), it follows from the condition and (22) that we have

$$
u(t)>0, \quad t \in[0,1] .
$$

By (23), we have

$$
\begin{aligned}
& u^{\prime}(0)=\frac{1}{\Gamma(\alpha)} \int_{0}^{1}(1-s)^{\alpha-1} d s>0, \\
& u^{\prime}(1) \leqslant \frac{1}{\Gamma(\alpha)} \int_{0}^{1}(1-s)^{\alpha-2}(2-s-\alpha) d s<0 .
\end{aligned}
$$

By (28), there exist $\varepsilon>0$ and $\tau_{1}>0$ such that

$$
\begin{aligned}
& u^{\prime}(t)>\tau_{1}, \quad t \in[0, \varepsilon], \\
& u^{\prime}(t)<-\tau_{1}, \quad t \in[1-\varepsilon, 1] .
\end{aligned}
$$

By (27), there exists $\tau_{2}>0$ such that $u(t)>\tau_{2}, t \in[\varepsilon, 1-\varepsilon]$. Letting $\tau_{3}=\min \left\{\tau_{1}, \tau_{2}\right\}$, for $v \in E,\|u-v\|<\tau_{3}$, we have

$$
\begin{aligned}
& v^{\prime}(t)>0, \quad t \in[0, \varepsilon], \\
& v^{\prime}(t)<0, \quad t \in[1-\varepsilon, 1], \\
& v(t)>0, \quad t \in[\varepsilon, 1-\varepsilon] .
\end{aligned}
$$

So, we have $v(t) \geqslant 0, \forall t \in[0,1]$. Therefore, $u \in P^{o}$. The proof is completed.

\section{Main Results}

In this section, we impose some growth conditions on $f$ which allow us to apply Theorems 5 and 6 to establish the existence of positive solutions to BVP (1).

Theorem 9. Assume $f:[0,+\infty) \times \mathbb{R} \rightarrow[0,+\infty)$ is continuous and there exist positive constants $C, k_{i}$ and $\sigma_{i} \in(0,1)(i=$ $1,2)$ such that

$\left(H_{1}\right) f(u, v) \leqslant C+k_{1}|u|^{\sigma_{1}}+k_{2}|v|^{\sigma_{2}}$ and $f(u, v) \not \equiv 0,(u, v) \epsilon$ $[0,+\infty) \times(-\infty,+\infty)$. Then BVP (1) has at least one positive solution.

Proof. Let $\bar{P}_{r}=\{u \in P,\|u\| \leqslant r\}$, where $r \geqslant$ $\max \left\{\left(3 k_{1} L\right)^{1 /\left(1-\sigma_{1}\right)},\left(3 k_{2} L\right)^{1 /\left(1-\sigma_{2}\right)}, 3 L C\right\}$, and $L=2 /(\alpha-$ 1) $\Gamma(\alpha)$. We now show that $A: \bar{P}_{r} \rightarrow \bar{P}_{r}$. In fact, if $u \in \bar{P}_{r}$, then $0 \leqslant u(t),\left|u^{\prime}(t)\right| \leqslant r, t \in[0,1]$. By condition $\left(H_{1}\right)$

$$
f(u, v) \leqslant C+k_{1} r^{\sigma_{1}}+k_{2} r^{\sigma_{2}} \text {. }
$$

By (25) and (31), we have

$$
\begin{aligned}
\left|(A u)^{\prime}(t)\right| & \leqslant \frac{2\left(C+k_{1} r^{\sigma_{1}}+k_{2} r^{\sigma_{2}}\right)}{(\alpha-1) \Gamma(\alpha)} \\
& =L\left(C+k_{1} r^{\sigma_{1}}+k_{2} r^{\sigma_{2}}\right), \quad t \in[0,1] ;
\end{aligned}
$$

this means $\|A u\| \leqslant L\left(C+k_{1} r^{\sigma_{1}}+k_{2} r^{\sigma_{2}}\right) \leqslant r$. By applying Schauder's fixed point theorem, the condition $f(u, v) \not \equiv$ 0 implies that $A$ has at least one nontrivial fixed point in $\bar{P}_{r}$, which is a positive solution of BVP (1). The proof is completed.

Theorem 10. Assume $f:[0,+\infty) \times \mathbb{R} \rightarrow[0,+\infty)$ is continuous and there exist two constants $R>r>0$ such that

$$
\begin{aligned}
& \left(H_{2}\right) f(u, v) \geqslant \alpha \Gamma(\alpha) r,(u, v) \in[0, r] \times[-r, r] ; \\
& \left(H_{3}\right) f(u, v) \leqslant(\alpha-1) \Gamma(\alpha) R / 2,(u, v) \in[0, R] \times[-R, R] .
\end{aligned}
$$

Then BVP (1) has at least one positive solution.

Proof. Take $\Omega_{1}=\{u \in P:\|u\|<r\}$; then, for $u \in \partial \Omega_{1}$, we have $0 \leqslant u(t),\left|u^{\prime}(t)\right| \leqslant r, t \in[0,1]$. From condition $\left(H_{2}\right)$, $f\left(u(t), u^{\prime}(t)\right) \geqslant \alpha \Gamma(\alpha) r, t \in[0,1]$. By (23), we have

$$
\begin{aligned}
\|A u\| & \geqslant\left|(A u)^{\prime}(0)\right|=\frac{\int_{0}^{1}(1-s)^{\alpha-1} f\left(u(s), u^{\prime}(s)\right) d s}{\Gamma(\alpha)} \\
& \geqslant \alpha r \int_{0}^{1}(1-s)^{\alpha-1} d s=r .
\end{aligned}
$$

Take $\Omega_{2}=\{u \in P:\|u\|<R\}$; then, for $u \in \partial \Omega_{2}$, we have $0 \leqslant u(t),\left|u^{\prime}(t)\right| \leqslant R, t \in[0,1]$. From condition $\left(H_{3}\right)$ and Lemma 4, we have

$$
\begin{aligned}
\left|(A u)^{\prime}(t)\right| \leqslant \frac{\int_{0}^{1} 2(1-s)^{\alpha-2} f\left(u(s), u^{\prime}(s)\right) d s}{\Gamma(\alpha)} & \\
\leqslant(\alpha-1) R\left(\int_{0}^{1}(1-s)^{\alpha-2} d s\right)=R, & t \in[0,1] .
\end{aligned}
$$


Consequently,

$$
\left\|(A u)^{\prime}\right\|_{0} \leqslant R
$$

It follows from (35) that $\|A u\| \leqslant R$ for $u \in \partial \Omega_{2}$. Therefore, by Theorem 5, $A$ has at least one fixed point in $P \cap\left(\bar{\Omega}_{2} \backslash\right.$ $\Omega_{1}$ ), which is the positive solution of BVP (1). The proof is completed.

To be convenient, we denote

$$
\begin{aligned}
& M=\max _{t \in[0,1]} \int_{0}^{1} G(t, s) d s, \\
& m=\max _{t \in[0,1]} \int_{1 / 4}^{3 / 4} G(t, s) d s, \\
& N=\frac{1}{\Gamma(\alpha)} \int_{0}^{1} 2(1-s)^{\alpha-2} d s .
\end{aligned}
$$

We will suppose that there are $R>r>\gamma r>r_{0}>0$ such that $f$ satisfies the following growth conditions:

$$
\begin{aligned}
& \left(H_{4}\right) f(u, v)<r_{0} / M,(u, v) \in\left[0, r_{0}\right] \times[-R, R] ; \\
& \left(H_{5}\right) f(u, v) \geqslant r / m,(u, v) \in[\gamma r, r] \times[-R, R] ; \\
& \left(H_{6}\right) f(u, v)<R / N,(u, v) \in[0, r] \times[-R, R],
\end{aligned}
$$

where $\gamma$ is defined by (18).

$$
\begin{aligned}
& f^{*}(u, v) \\
& = \begin{cases}f(u, v), & (u, v) \in[0, r] \times(-\infty,+\infty), \\
f(r, v), & (u, v) \in(r,+\infty) \times(-\infty,+\infty),\end{cases} \\
& \tilde{f}(u, v) \\
& = \begin{cases}f^{*}(u, v), & (u, v) \in[0,+\infty) \times[-R, R], \\
f^{*}(u,-R), & (u, v) \in[0,+\infty) \times(-\infty,-R), \\
f^{*}(u, R), & (u, v) \in[0,+\infty) \times(R,+\infty) .\end{cases}
\end{aligned}
$$

$$
\begin{aligned}
\beta(\widetilde{A} u) & =\frac{\max _{t \in[0,1]}\left|\int_{0}^{t}\left[(1-s)^{\alpha-1}-(\alpha-1)(t-s)^{\alpha-2}\right] \tilde{f}\left(u(s), u^{\prime}(s)\right) d s+\int_{t}^{1}(1-s)^{\alpha-1} \tilde{f}\left(u(s), u^{\prime}(s)\right) d s\right|}{\Gamma(\alpha)} \\
& \leqslant \frac{\max _{t \in[0,1]}\left|\int_{0}^{1} 2(1-s)^{\alpha-2} \tilde{f}\left(u(s), u^{\prime}(s)\right) d s\right|}{\Gamma(\alpha)}<\frac{2 R \int_{0}^{1}(1-s)^{\alpha-2} d s}{N \Gamma(\alpha)}=R .
\end{aligned}
$$

Then $\tilde{f}(u, v)=f(u, v)$ for $(u, v) \in[0, r] \times[-R, R]$. Define

$$
\widetilde{A} u(t)=\int_{0}^{1} G(t, s) \tilde{f}\left(u(s), u^{\prime}(s)\right) d s .
$$

Theorem 11. Assume $f:[0,+\infty) \times \mathbb{R} \rightarrow[0,+\infty)$ is continuous and conditions $\left(H_{4}\right)-\left(H_{6}\right)$ hold. Then BVP (1) has at least one positive $u_{0}$ satisfying $\alpha\left(u_{0}\right) \in\left(r_{0}, r\right), \beta\left(u_{0}\right)<R$.

Proof. Take

$$
\begin{aligned}
& \Omega_{1}=\left\{u \in E: \alpha(u)<r_{0}, \beta(u)<R\right\}, \\
& \Omega_{2}=\{u \in E: \alpha(u)<r, \beta(u)<R\}, \\
& D_{1}=\left\{u \in E: \alpha(u)=r_{0}\right\}, \\
& D_{2}=\{u \in E: \alpha(u)=r\} .
\end{aligned}
$$

Since $f:[0,+\infty) \times \mathbb{R} \rightarrow[0,+\infty)$ is continuous, by Lemma 7 and (37)-(38), it is obvious that $\widetilde{A}: P \rightarrow P$ is also completely continuous, and there is a $w \in\left(\Omega_{2} \cap P\right) \backslash\{\theta\}$ such that $\alpha(u+$ $\lambda w) \geqslant \alpha(u), u \in P, \lambda \geqslant 0$. For $u \in D_{1} \cap P$, then $0 \leqslant u(t) \leqslant r_{0}$, $t \in[0,1]$. From condition $\left(H_{4}\right)$, we have

$$
\begin{aligned}
\alpha(\widetilde{A} u) & =\max _{t \in[0,1]}\left|\int_{0}^{1} G(t, s) \tilde{f}\left(u(s), u^{\prime}(s)\right) d s\right| \\
& <\frac{r_{0}}{M} \max _{t \in[0,1]} \int_{0}^{1} G(t, s) d s=r_{0}
\end{aligned}
$$

whereas, for $u \in D_{2} \cap P$, according to the definition of $P$ that $\gamma r=\gamma\|u\|_{0} \leqslant u(t) \leqslant r, t \in[1 / 4,3 / 4]$. From condition $\left(H_{5}\right)$, we have

$$
\begin{aligned}
\alpha(\widetilde{A} u) & =\max _{t \in[0,1]}\left|\int_{0}^{1} G(t, s) \tilde{f}\left(u(s), u^{\prime}(s)\right) d s\right| \\
& >\max _{t \in[0,1]}\left|\int_{1 / 4}^{3 / 4} G(t, s) \tilde{f}\left(u(s), u^{\prime}(s)\right) d s\right| \\
& \geqslant \frac{r}{m} \max _{t \in[0,1]} \int_{1 / 4}^{3 / 4} G(t, s) d s=r .
\end{aligned}
$$

Finally, for $u \in P$, from condition $\left(H_{6}\right)$ and Lemma 4 , we have
According to Theorem $6, \widetilde{A}$ has at least one fixed point $u_{0} \in$ $P \cap\left(\Omega_{2} \backslash \bar{\Omega}_{1}\right)$. Noticing that $\tilde{f}(u, v)=f(u, v)$ for $u \in \Omega_{2}$, we 
We now present some simple examples. Consider the following (BVP):

$$
\begin{aligned}
{ }^{C} D_{0+}^{5 / 2} u(t)+f\left(u(t), u^{\prime}(t)\right) & =0, \quad t \in(0,1), \\
u(0) & =u(1)=u^{\prime \prime}(0)=0 .
\end{aligned}
$$

Example 12. Let $f(u, v)=1+u^{1 / 2}+|v|^{1 / 2},(u, v) \in[0,+\infty) \times \mathbb{R}$. It is clear that $f$ satisfies all the conditions of Theorem 9. Then, by Theorem 9, BVP (43) has at least one positive solution.

Example 13. Let $f(u, v)=3.5+u / 2+v^{2} / 100,(u, v) \epsilon$ $[0,+\infty) \times \mathbb{R}$. Choosing $R=10, r=1$, then $f$ satisfies all the conditions of Theorem 10. By Theorem 10, BVP (43) has at least one positive solution.

Example 14. Let $f(u, v)=1800 u^{3}+1 /\left(v^{2}+1\right),(u, v) \in$ $[0,+\infty) \times \mathbb{R}$. By simple calculation, we have $M^{-1} \approx 10.23$, $m^{-1} \approx 17.58, N^{-1} \approx 1$. Choosing $R=2000, r=1$, and $r_{0}=$ 0.05 , it is easy to verify that $f$ satisfies all the conditions of Theorem 11. By Theorem 11, BVP (43) has at least one positive solution.

\section{Conflict of Interests}

The authors declare that there is no conflict of interests regarding the publication of this paper.

\section{Authors' Contribution}

Shugui Kang conceived the study and participated in its design. Yaqiong Cui drafted the paper and participated in its design and coordination. Zhiping Liu participated in the sequence correction. All authors read and approved the final paper.

\section{Acknowledgments}

The authors are very grateful to the reviewers for their valuable suggestions and useful comments, which led to an improvement of this paper. The project is supported by National Nature Science Foundation of China (11271235) and The Foundation of Datong University (2013K5).

\section{References}

[1] Z. Bai and H. Lü, "Positive solutions for boundary value problem of nonlinear fractional differential equation," Journal of Mathematical Analysis and Applications, vol. 311, no. 2, pp. 495-505, 2005.

[2] X. Xu, D. Jiang, and C. Yuan, "Multiple positive solutions for the boundary value problem of a nonlinear fractional differential equation," Nonlinear Analysis, vol. 71, no. 10, pp. 4676-4688, 2009.

[3] Z. Bai, "Eigenvalue intervals for a class of fractional boundary value problem," Computers \& Mathematics with Applications, vol. 64, no. 10, pp. 3253-3257, 2012.
[4] X. Zhang, L. Wang, and Q. Sun, "Existence of positive solutions for a class of nonlinear fractional differential equations with integral boundary conditions and a parameter," Applied Mathematics and Computation, vol. 226, pp. 708-718, 2014.

[5] X. Zhang, "Positive solutions for a class of singular fractional differential equation with infinite-point boundary value conditions," Applied Mathematics Letters, vol. 39, pp. 22-27, 2015.

[6] A. Guezane-Lakoud and R. Khaldi, "Solvability of a fractional boundary value problem with fractional integral condition," Nonlinear Analysis, vol. 75, no. 4, pp. 2692-2700, 2012.

[7] A. Cabada and G. Wang, "Positive solutions of nonlinear fractional differential equations with integral boundary value conditions," Journal of Mathematical Analysis and Applications, vol. 389, no. 1, pp. 403-411, 2012.

[8] X. Yang, Z. Wei, and W. Dong, "Existence of positive solutions for the boundary value problem of nonlinear fractional differential equations," Communications in Nonlinear Science and Numerical Simulation, vol. 17, no. 1, pp. 85-92, 2012.

[9] Y. Li, "Positive solutions of fourth-order boundary value problems with two parameters," Journal of Mathematical Analysis and Applications, vol. 281, no. 2, pp. 477-484, 2003.

[10] Y. Guo and W. Ge, "Positive solutions for three-point boundary value problems with dependence on the first order derivative," Journal of Mathematical Analysis and Applications, vol. 290, no. 1, pp. 291-301, 2004.

[11] D. Guo, Nonlinear Functional Analysis, Shandong Science \& Technology Press, 2nd edition, 2001 (Chinese).

[12] K. B. Oldham and J. Spanier, The Fractional Calculus, Academic Press, New York, NY, USA, 1974. 


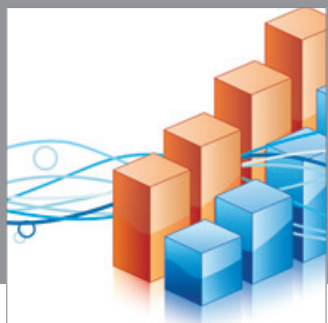

Advances in

Operations Research

mansans

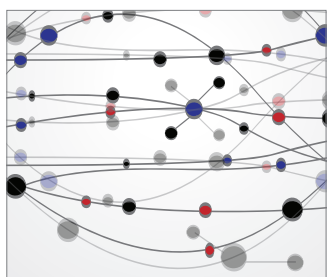

The Scientific World Journal
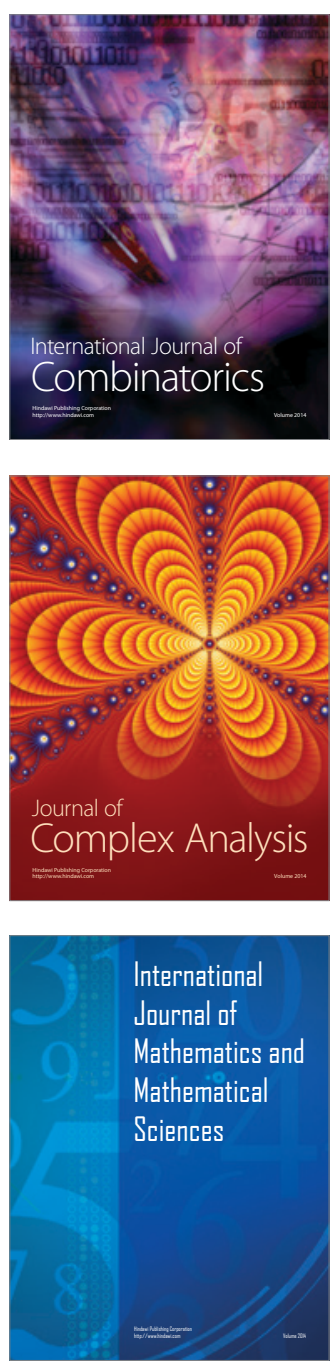
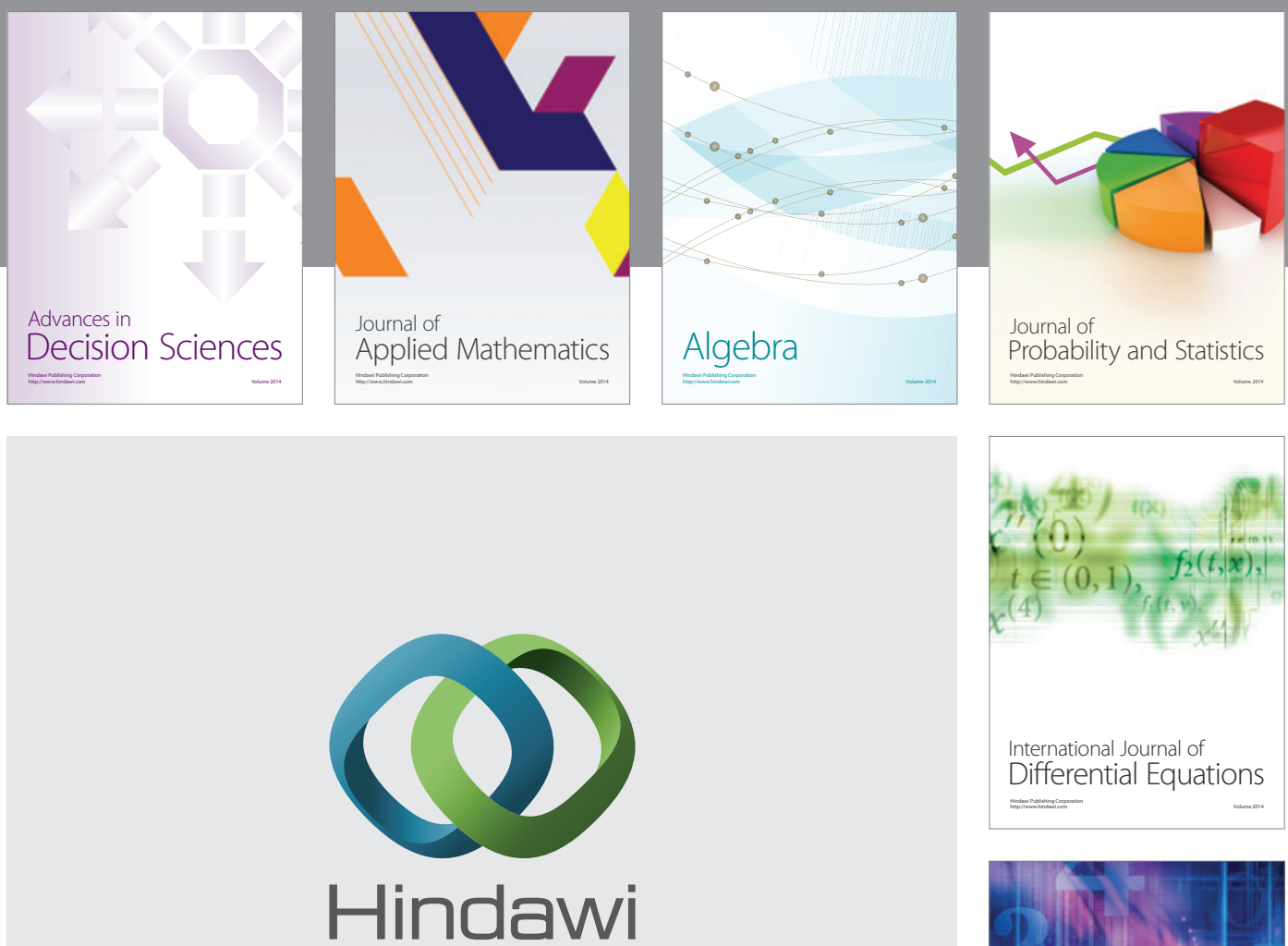

Submit your manuscripts at http://www.hindawi.com
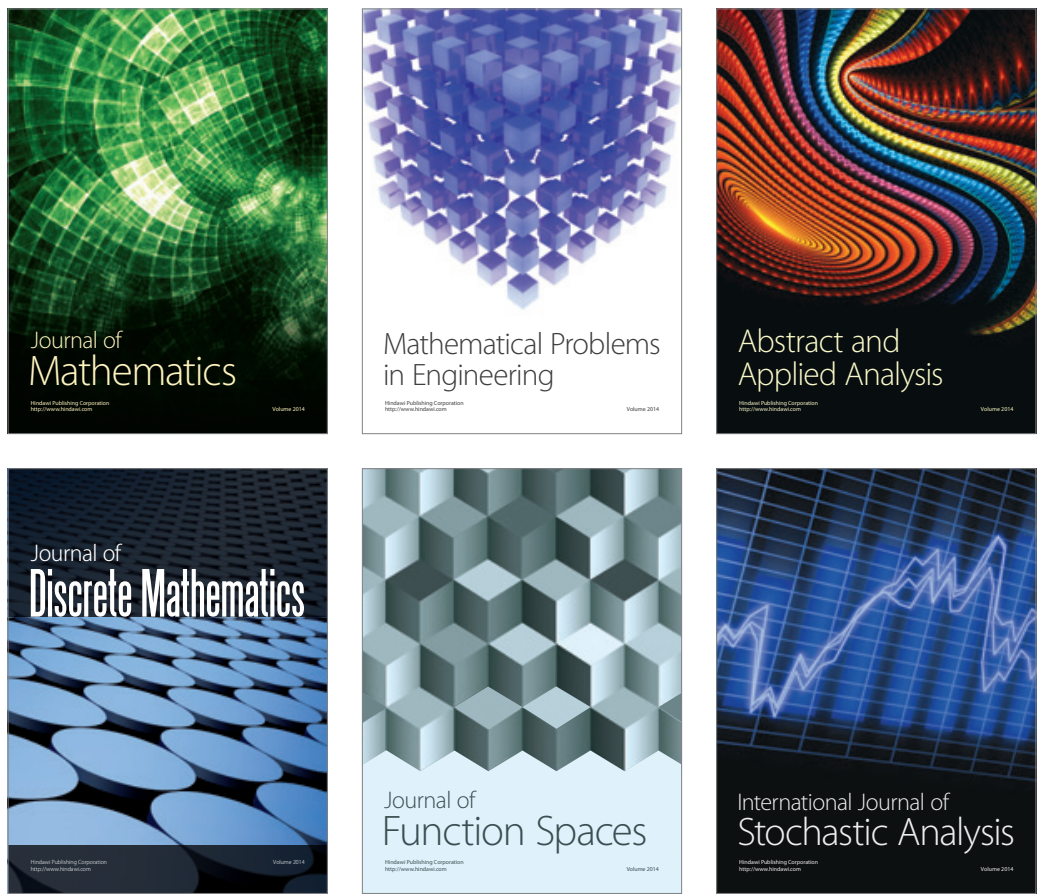

Journal of

Function Spaces

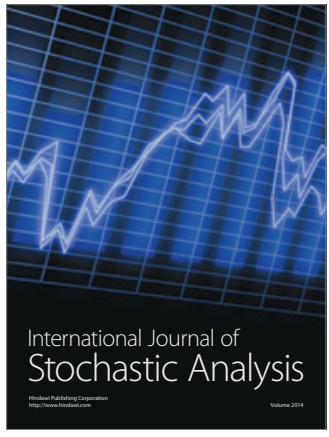

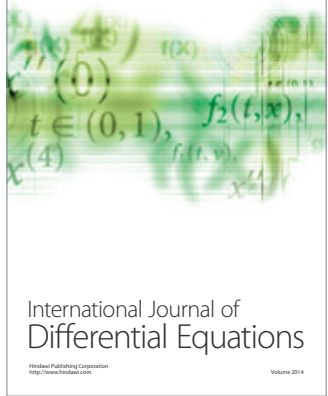
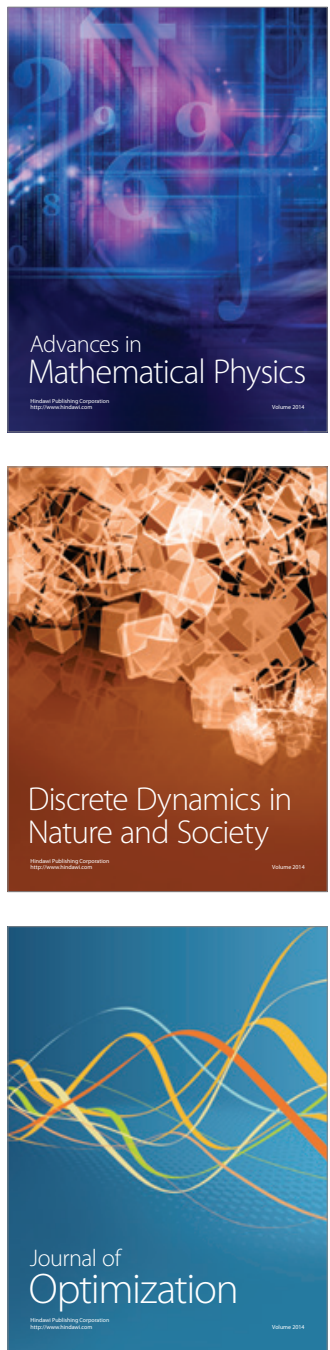